\title{
A ESPERA NA VIDA URBANA: UMA ANÁLISE PSICOSSOCIAL DAS FILAS ${ }^{1}$
}

\author{
Fabio Iglesias \\ Hartmut Günther"
}

\begin{abstract}
RESUMO. A fila de espera assume inúmeras funções na alocação dos recursos na vida urbana, como espaço, tempo, serviços e produtos. Suas configurações variam desde a típica fila presencial, aquelas em que as pessoas aguardam em pé umas atrás das outras, até formas implícitas de organização da espera, como aquelas geradas por tecnologias de comunicação remota providas pelo telefone e pela internet. Este artigo aborda a fila de espera como um sistema social, focando sua configuração presencial e destacando o fato de que, embora seja um fenômeno ubíquo, verifica-se certa ausência de estudos sobre esse tema na psicologia social. Pretende-se, assim: (a) analisar as características gerais das filas; (b) apresentar uma breve revisão do conceito de fila nas ciências exatas, biológicas e sociais aplicadas; (c) revisar estudos sobre filas na psicologia social; e (d) apontar maneiras de como a fila pode ser tanto tema quanto palco de estudos da psicologia.
\end{abstract}

Palavras-chave: Fila de espera, sistema social, ambiente urbano.

\section{WAITING AND URBAN LIFE: A SOCIAL PSYCHOLOGICAL ANALYSIS OF LINES}

\begin{abstract}
Waiting lines (queues) assume various functions in the allocation of urban resources, such as access to space, time, services or products. They vary from the typical line where people are standing one behind the other to such virtual ones as in telecommunication or the Internet. The paper looks at queues as social systems, and focuses on the presential configuration, paying special attention to the fact that, while ubiquitous, queues are not receiving much attention in the social psychological literature. Thus this article (a) analyzes the general features of queues, (b) offers a short review of the concept of queues in the formal, biomedical and applied social sciences, (c) reviews the social psychological literature about queues, (d) indicates how queues may serve both as a topic of research, as well as an environmental scenario for research in psychology.
\end{abstract}

Key words: Waiting line/queue, social system; urban environment.

\section{LA ESPERA EN LA VIDA URBANA: UN ANÁLISIS PSICOSOCIAL DE LAS COLAS}

RESUMEN. Son inúmeras las funciones que asume la cola de espera en la asignación de recursos urbanos, como espacio, tiempo, servicios y productos. Las configuraciones pueden variar desde la típica cola presencial - aquellas en que la gente aguarda de pie - hasta formas implícitas de organización de la espera - las generadas por tecnologías de comunicación remota proveídas por teléfono e internet. Este artículo aborda la cola de espera como un sistema social, fijándose en su configuración presencial y destacando que, aunque sea un fenómeno ubicuo, la psicología social ha tratado del tema de forma muy escasa. Así, se pretende (a) analizar las características generales de las colas, (b) presentar un breve repaso del concepto de cola en las ciencias exactas, biomédicas y sociales aplicadas, (c) rever estudios sobre colas en la psicología social e (d) indicar maneras de tratar la cola como tema y escenario de estudio de la psicología.

Palabras-clave: Colas de espera, sistema social, ambiente urbano.

A fila de espera cumpre uma função de organização social indissociável da vida urbana. Assim como o surgimento da psicologia social moderna, o interesse pela fila pode ser relacionado aos efeitos da revolução industrial a partir do século XVIII, que mudou significativamente o cenário da

\footnotetext{
Apoio: CNPq.

* Doutor em Psicologia. Professor do Programa de Pós-graduação em Psicologia Social, do Trabalho e das Organizações da Universidade de Brasília.

\# PhD em Psicologia Social pela University of California at Davis, Professor Titular do Programa de Pós-graduação em Psicologia Social, do Trabalho e das Organizações da Universidade de Brasília.
} 
civilização, passando a concentrar grande número de pessoas nas metrópoles (Simmel, 1903/1987). Considerando-se que quanto maior a densidade populacional maior é a competição pelos recursos que as cidades podem oferecer, a demanda maior que a oferta implica no surgimento de filas de espera, o que justifica o interesse pelo tema. Schwartz (1978) foi adiante neste argumento, mostrando que toda a sociedade pode ser analisada como uma grande rede de filas inter-relacionadas. Esta rede serve como metáfora para descrever a dependência para com o tempo e a sua alocação perante o espaço e como se criam os agendamentos e os sequenciamentos dos movimentos. Filas de espera constituem, portanto, uma etapa inevitável para acesso a um sem-número de produtos e serviços, e até mesmo para o simples transitar no espaço.

Ao mesmo tempo em que a fila é ubíqua e assume diversas configurações como fenômeno social por excelência, verifica-se certa ausência da fila como objeto de estudo na literatura da psicologia social. Deste modo, o propósito deste trabalho é: (a) analisar as principais características das filas de espera; (b) apresentar uma breve revisão do conceito de fila nas ciências exatas, biomédicas e sociais aplicadas; (c) revisar estudos sobre fila na psicologia social; e (d) apontar maneiras de como a fila pode ser tanto tema quanto palco de estudos da psicologia. Apresentamos inicialmente, desta maneira, duas classificações de filas de espera, para em seguida analisá-las como objeto de estudo da psicologia social.

\section{CARACTERÍSTICAS GERAIS DAS FILAS}

Uma primeira distinção a ser feita é entre a fila de espera presencial e a não-presencial. A primeira exige comportamento específico, como a permanência em pé ou sentado, geralmente atrás dos que chegaram antes. Podem-se incluir nessa primeira categoria as filas organizadas por agendamento prévio ou por sistemas de senhas que, embora presenciais para o atendimento, liberam o usuário para realizar outras atividades, já que não precisa permanecer em determinada posição para garantir a prioridade. Nestes casos o usuário pode até se ausentar do local por tempo determinado. Estando presente, o usuário pode observar a qualidade do atendimento, acompanhar o andamento da fila ou, pelo número da senha, ter uma estimativa de seu tempo de espera.

A fila não-presencial é invisível, sem a presença física do usuário. Frequentemente o usuário nem sequer tem informação sobre o processo de atendimento, o tempo previsto de espera ou o número de pessoas à sua frente. Tal é o caso de serviços como solicitações por telefone e, mais recentemente, pela Internet, chamados por Zohar, Mandelbaum e Shimkin (2002) de "telefilas". O foco principal desta parte do trabalho é a fila presencial, tendo em vista os comportamentos humanos envolvidos e as interações sociais decorrentes.

\section{FILA ÚNICA E FILAS MÚLTIPLAS}

Existem duas maneiras de organizar a fila presencial: em fila única e em filas múltiplas. A fila única é aquela em que os usuários formam uma única sequência, posicionando-se uns atrás dos outros conforme a ordem de chegada. Pode haver mais de um guichê de atendimento, mas o acesso a qualquer um deles é definido de maneira quase randômica, dependendo do momento em que o usuário chegue ao início da fila. As filas múltiplas são aquelas que se formam individualmente para cada guichê de atendimento. Desta maneira, a ordem de atendimento depende da ordem de chegada numa determinada fila, não da ordem de chegada ao ambiente do serviço. Não existe consenso sobre que tipo de fila melhor atende o usuário, tanto do ponto de vista do sentimento de justiça experienciado pelas pessoas envolvidas (Larson \& Odoni, 1981; Rafaeli, Barron \& Haber, 2002) quanto do ponto de vista dos custos e benefícios gerenciais (Cooper, 1981; Rothkopf \& Rech, 1987).

Jones, O’Berski e Tom (1980) apontam que, mesmo que a fila única diminua objetivamente o tempo médio de espera em muitas situações, ela não oferece liberdade ao usuário para selecionar o balcão de atendimento ou o atendente que tenha despertado alguma preferência específica. Ao organizar o sistema de filas, o gestor deve considerar a possibilidade de o consumidor preferir ter algum controle sobre a escolha da fila. Além disso, há usuários que preferem poder comparar o tamanho e andamento de sua fila com as filas ao lado, para poder trocar a fila caso estimem que aquela ao lado anda mais rápido, como ocorre em situações de congestionamento no trânsito.

Por outro lado, a fila múltipla pode gerar um senso de injustiça, quando o usuário conclui que a fila ao lado anda mais rápido, mesmo que isso não seja verdade. Embora o sistema de filas múltiplas não garanta a prioridade por ordem de chegada ao ambiente de serviço, a liberdade de escolha da fila pode fazer com que o usuário avalie a fila múltipla como melhor. Rafaeli at al. (2002), entretanto, verificaram que usuários preferem a fila única mesmo quando o tempo de espera é igual em ambas as filas. Referindo-se a um "paradoxo da fila", verificaram 
ainda que os usuários mantêm essa preferência mesmo quando o tempo de espera na fila única se torna maior.

Oportunamente, o dicionário oferece uma definição de fila que contempla aspectos negligenciados pela psicologia social. A fila é definida como uma "fileira de pessoas que se colocam umas atrás das outras, pela ordem cronológica ou de chegada a um ponto de embarque em veículos urbanos, a guichês ou a quaisquer estabelecimentos onde haja grande afluência de interessados" (Ferreira, 1986). Três aspectos dessa definição distinguem a abordagem psicológica aqui proposta do modo como as filas têm sido investigadas nas ciências anteriormente descritas.

Em primeiro lugar, a fila de espera é definida como presencial, constituída por pessoas que se comportam, que têm características e diferenças individuais, sendo que as possibilidades de análise dessas variáveis são perdidas ao se tratar a fila como uma mera expressão de uma função aritmética. Isso implica que a fila presencial ocorre em algum ambiente específico, como organização espacial concreta, portanto sujeita a uma série de variáveis situacionais imediatas. Assim, a fila não é somente um conceito abstrato de representação de pessoas esperando por atendimento.

Um segundo aspecto refere-se a normas sociais desse tipo de organização, visto que filas geralmente se formam de modo a priorizar o atendimento de seus usuários pela ordem de chegada. Raramente esse tipo de regra é explícito ou previsto legalmente, embora haja leis que garantem prioridade para casos especiais, de modo que os comportamentos em fila são muito mais regulados por normas socialmente compartilhadas. Essas normas dependem mais uma vez de variáveis situacionais, do grau de relação e conhecimento que os usuários mantêm entre si, de aspectos culturais de ordem maior e de questões relacionadas a justiça e atribuição de causalidade.

Finalmente, a definição destaca a situação de grande afluência de interessados como condição para o surgimento da fila. Demanda maior que a oferta é a melhor expressão para descrever a necessidade de organizar pessoas esperando por atendimento, isto é, quando não há como suprir num mesmo momento a necessidade de todos os interessados. Trata-se, mais uma vez, de um aspecto psicológico, ressaltando-se que fila implica no surgimento de grupos, na disputa de recursos e nos comportamentos pró-sociais ou antissociais que podem advir dessa situação.

O dicionário condiz, assim, com a síntese de Milgram, Liberty, Toledo e Wackenhut (1986) da fila presencial a partir dos trabalhos de psicologia social sobre o tema. Sua função é regular a sequência pela qual as pessoas acessam bens ou serviços. Sua ordenação tem uma forma espacial distintiva e sua manutenção depende de um conhecimento compartilhado de padrões de comportamento apropriados à situação.

\section{A FILA NAS CIÊNCIAS EXATAS, BIOMÉDICAS E SOCIAIS APLICADAS}

A maior parte dos estudos sobre filas foi iniciada para solucionar problemas práticos decorrentes do avanço tecnológico e da alta concentração urbana. Existem concepções de fila de espera claramente distintas conforme suas características abordadas e conforme seja tratada como algo abstrato ou concreto. Essas concepções têm lugar em campos diversos: nas ciências exatas, como na matemática e na engenharia; nas ciências biomédicas e nas ciências sociais, assim como no marketing, na sociologia e na psicologia.

Ao desenvolvimento das comunicações telefônicas é atribuída a origem da área matemática chamada "teoria das filas" (Cooper, 1981), também conhecida como teoria da congestão. O uso do telefone gerou a necessidade de se conhecer o número ótimo de circuitos e operadores para lidar com o congestionamento de ligações, considerando-se a relação entre a flutuação da demanda pelo serviço ao longo do tempo e a espera que deve ser vivenciada por seus usuários. Primeiramente elaborada em 1909 por Agner K. Erlang, engenheiro-chefe, na Dinamarca, da International Bell Telephone Company, a teoria das filas se consolidou como um ramo da teoria da probabilidade aplicada, tratando a fila como um processo de ordenação que inclui variáveis como o tipo e o número de filas envolvidas, o número de servidores e o tempo médio de espera, além de as probabilidades do tempo de espera e a demanda aumentarem ou diminuírem. As aplicações da teoria das filas expandiram-se para diversas outras áreas, entre as quais se destacam a engenharia de produção e a engenharia de tráfego, mas os modelos raramente consideram variáveis psicológicas que determinem comportamentos diferenciados dos indivíduos envolvidos, representando-os basicamente por meio de expressões numéricas.

Essas ciências são responsáveis pelo estudo da disciplina da fila, ou seja, do princípio que define a ordem de atendimento e suas consequências práticas. Filas geralmente são definidas pela regra simplificada na expressão first come, first served ("chegou primeiro, atendido primeiro"), o modo principal de 
gerenciar o atendimento em função da ordem de chegada, garantindo maior equidade aos usuários (Cooper, 1981). Existem, poré, outras regras, tais como last in, first out ("chegou por último, sai primeiro"), shortest service time ("tarefa menor é realizada primeiro") ou até service in random order ("ordem randômica de atendimento"), isto é, a loteria pode ser preferida como sistema de alocação de recursos.

A teoria das filas tem um papel central na análise e planejamento de serviços urbanos, entretanto trabalha com simplificações e aproximações. Do ponto de vista da psicologia essa análise é incompleta, pois o comportamento humano está sob influência direta de variáveis contextuais de difícil predição. Nota-se que as estimativas baseadas na teoria das filas frequentemente se originam mais em intuição e experiência do que em evidências empíricas, justamente para tornar viáveis os modelos de predição (Larson \& Odoni, 1981).

No âmbito das ciências biomédicas a necessidade de organizar o acesso a serviços médico-hospitalares escassos deu origem a diversos estudos sobre filas de espera, destacando-se o problema das filas para transplantes de órgãos (Cullis \& Jones, 1985). O fato de os órgãos humanos potencialmente transplantáveis terem sobrevida curta torna o gerenciamento da fila especialmente complexa. A fila é tratada como uma abstração, pois aos pacientes são atribuídos números numa ordenação que não é fixa, mas ainda varia conforme a disponibilidade, a gravidade da demanda, as distâncias existentes entre as partes envolvidas e características de compatibilidade entre doador e receptor. As filas presenciais no atendimento ambulatorial, clínico e hospitalar parecem ter menor destaque na literatura da área, embora constituam um problema muito mais imediato.

Já na administração e no marketing a fila é tratada como uma organização concreta de pessoas, sendo os estudos considerados pelo seu valor prático e suas implicações gerenciais. Esses estudos destacam diferenças individuais, mas pouco revelam sobre a dinâmica do comportamento de usuários em filas, algo que só é verificado nos estudos das ciências sociais e, em especial, na psicologia.

A estimativa de tempo pelo usuário é uma das principais linhas de investigação sobre filas nas ciências sociais aplicadas, já que é um dos fatores determinantes na avaliação da qualidade dos serviços e produtos. Estas análises diferem dos estudos da teoria das filas, que investigam o tempo de espera pelo atendimento enquanto objetivamente mensurável. Assim, administradores procuram gerenciar a percepção do tempo de espera pelo usuário por meio do sistema de atendimento, dos recursos do ambiente e da expectativa sobre o produto ou serviço desejado (Cogan, 1998; Maister, 1985). Destaca-se, entretanto, que filas também podem ser bem avaliadas e vistas como sinal de atratividade, quando alta demanda sinaliza status ou qualidade, como em restaurantes e consultórios médicos, aspecto amplamente explorado na área (Kostecki, 1996): quanto maior a espera, melhor seria a avaliação do produto ou do serviço, justificando o comportamento de esperar.

\section{A ATENÇÃo ÀS FILAS DE ESPERA NA LITERATURA PSICOSSOCIAL}

Constata-se que o tema filas de espera não tem recebido muita atenção nas ciências humanas, ainda que já se tenha acumulado um suporte empírico para mostrar que é mais do que uma mera organização espacial ou um problema de matemática (Iglesias \& Günther, 2007). O exame de alguns dos livros-texto de psicologia social mais conhecidos em língua portuguesa, sejam eles originais (por exemplo: Rodrigues, Assmar \& Jablonski, 2000; Vala \& Monteiro, 2004) ou traduzidos para o português (por exemplo: Aronson, Wilson \& Akert, 2003; Myers, 2000), revela que o termo "fila" não aparece no índice remissivo de nenhum deles, sendo que a maioria nem sequer faz referência a pesquisas específicas sobre o tema.

Constatação similar é possível nas cinco edições do Handbook of Social Psychology (destacadamente, Gilbert, Fiske \& Lindzey, 1998), que não têm em seus índices remissivos os termos queue, termo técnico, ou waiting line, expressão cotidiana, apesar de Milgram e Toch (1969) terem dedicado um capítulo inteiro da segunda edição do Handbook a movimentos coletivos, incluindo neles o fenômeno das filas de espera. A situação não difere ao se verificar o índice geral dos seis volumes de La Psychologie Sociale presente no primeiro deles (Beauvois, 1995). Esta tendência continua em livros-textos de língua inglesa, como os de Smith e Mackie (1995) e ainda Baron e Byrne (2003), o livro de psicologia social mais vendido no mundo.

Há que destacar duas publicações que dedicaram certa atenção ao problema do tempo de espera e das filas, frutos de investigação psicossocial empírica, dirigidas a um público mais amplo. Schwartz (1975) examinou os fenômenos de poder, de interação social e da justiça envolvidos em filas de espera, sendo, até hoje, o único trabalho sistemático sobre esse tema. Tornou-se um clássico de psicologia social e 
sociologia urbana, com valor heurístico na geração de hipóteses sobre diversos fenômenos. Levine (1997) examinou diferenças culturais associadas ao significado do tempo, reunindo experiências pessoais e estudos empíricos em vários países. Dedicou um capítulo à relação entre tempo e poder, com breve revisão da literatura sobre filas de espera, que inclui estudos sobre tolerância à espera e pontualidade, atribuição de causas à demora no atendimento e reações a intrusos.

Quanto aos artigos em periódicos em português, a busca pelo termo "fila(s) de espera" nas bases de dados Scielo no Brasil e em Portugal (www.scielo.br e www.scielo.oces.mctes.pt) e PEPsic (www.bvspsi.org.br) não retorna atualmente nenhum resultado. O termo "filas" retorna apenas referências na área de engenharia de produção, tratando o tema sob uma perspectiva operacional, ou então sob perspectivas biomédicas e econômicas. Os termos "tempo" e "tempo de espera" tampouco retornam qualquer referência significativa.

No cenário internacional verifica-se que os periódicos indexados em psicologia oferecem poucos artigos específicos sobre o tema. A busca na base de dados PsycINFO com o termo "waiting line" retorna doze artigos, sendo que apenas quatro foram publicados em revistas de psicologia, com o foco em filas no sentido aqui analisado. Ainda assim, nem sempre os fenômenos foram investigados em filas reais, mas sim, em cenários fictícios e vídeos apresentados em laboratório. A busca pelo termo "quеие", que tem um significado mais amplo, retorna 73 artigos, já incluindo aqueles classificados pelo termo "waiting line" acima referidos, mas apenas onze deles têm uma abordagem social e poucos investigaram filas reais, já citados na revisão acima, pois a maior parte se concentra no uso de cenários, simulações em computador e fotografias ou são estudos puramente teóricos.

Em suma, como objeto de estudo, a fila sofre de subestimação semelhante à alertada por Rozestraten (1988) em relação ao estudo do comportamento no trânsito. Este tende a ser visto mais como um meio do que um fim, razão pela qual por longo tempo esteve ausente da pesquisa científica. Cumpre observar que a fila também é um problema de trânsito, isto é, um meio de acesso, e raramente um fim em si; mas, a fila de espera constitui um tipo singular de sistema sócia,1 com normas, valores e papéis diferenciados (Mann, 1969, 1977; Mann \& Taylor, 1969; Milgram et al., 1986; Schmitt e cols., 1992; Schwartz, 1975). Nela podem ser investigados inúmeros fenômenos psicológicos de outras subáreas além da psicologia social, como a psicologia ambiental e a psicologia do consumidor.

\section{As pesquisas empíricas}

Milgram et al. (1986) observaram que até a década de 1960 a psicologia social estudou a interação social em díades e pequenos grupos, não examinando como as pessoas são afetadas pelo ambiente urbano. Com o surgimento da psicologia ambiental mencionando-se aqui apenas um trabalho importante do próprio Milgram sobre a vida na cidade (1970) - o ambiente começou a se a destacar novamente como elemento importante da análise do comportamento social, consolidando temas que são hoje essenciais à identidade da psicologia ambiental. Os estudos sobre filas surgem do ponto de vista da organização e regulação do acesso a serviços com o princípio de igualdade quando não há como atender ao mesmo tempo certo número de pessoas, protegendo assim a prioridade das que chegaram antes.

Antes dos trabalhos empíricos, a fila foi tratada como um "fenômeno de massa". Brown (1954) propôs uma taxonomia dos tipos de comportamento coletivo, classificando a fila entre os "tumultos urbanos". Essa classificação acompanhou o conceito prevalente no início do século XX, quando comportamentos de massa eram vistos como irracionais, desordenados e instáveis. Desta maneira, a concepção de fila da época sofre a influência de Le Bon (1895/1954). Essa visão do comportamento social irracional foi perpetuada por psicólogos influentes como Floyd Allport, Neil Miller e John Dollard durante a primeira metade do século $\mathrm{XX}$, até que trabalhos experimentais sobre conflito e cooperação fundamentaram o início de um debate que se estende até hoje sobre a racionalidadeirracionalidade da multidão (Adamatzky, 2005; Surowiecki, 2004).

Mann (1969) foi um dos primeiros autores na psicologia social a combater de maneira sistemática essa visão irracional, rejeitando estereótipos negativos sobre as massas e sobre a própria fila. Ao mesmo tempo em que Milgram e Toch (1969) salientaram a natureza racional da multidão, os trabalhos de Mann foram pioneiros em investigar a fila como um fenômeno social e racional distinto na psicologia. Mann estudou a dinâmica da fila e os comportamentos observáveis nela, suas regularidades e seu significado cultural, mostrando que filas são guiadas muito mais por comportamentos cooperativos e adaptativos do que pelos competitivos e egoístas enfatizados na abordagem de Brown (1954). Mann (1970) insistiu que a fila mostra a capacidade de uma massa amorfa gerar um microssistema social organizado e inibitório 
de conflitos, constituindo-se na mais ordenada, cooperativa e estruturada forma de comportamento coletivo.

$\mathrm{Na}$ psicologia, os primeiros trabalhos empíricos sobre filas espontâneas em campo de que se tem conhecimento foram publicados por Mann (1969) e Mann \& Taylor (1969). Leon Mann iniciou um programa de pesquisas sobre o tema na década de 1960, encerrado no final da década de 1970, valendose de experiência intercultural em Israel, nos Estados Unidos e na Austrália. Seus primeiros artigos foram inovadores para o tema na psicologia social, pois mostraram as possibilidades de estudo que a fila oferece, tanto em nível micro das interações sociais quanto em seu significado cultural. Investigou filas que se formaram dias antes do início de venda de ingressos para um campeonato de futebol australiano, em que centenas de pessoas pernoitavam e, mesmo completamente desconhecidas, acabavam constituindo grupos informais que se ajudavam mutuamente e zelavam pela manutenção da ordem na fila. Mann mostrou também como certos comportamentos específicos, como tentativas de intrusão e de guardar lugar na fila, eram determinados pelo tamanho da fila, pela posição dos usuários e pelas expectativas individuais de sucesso na obtenção de ingressos escassos. O mesmo conteúdo desse artigo apareceu para uma audiência mais ampla (Mann, 1970), despertando atenção do público para um problema urbano que se mostrava cada vez mais prevalente e merecedor de investigação científica, ao lado de um interesse crescente pelas modificações no comportamento humano exercidas pelas grandes cidades modernas (Milgram, 1970).

Mann e Taylor (1969) também realizaram estudo empírico sobre como pessoas em diferentes posições na fila estimam o número de pessoas à frente, encontrando tendências que puderam ser confirmadas no contexto brasileiro (Iglesias et al. 2004). Mann e Taylor interessaram-se pelo modo como pessoas no início da fila tendem a superestimar o tamanho da fila adiante, enquanto pessoas em seu final tendem a subestimá-lo, erros interpretados como formas de justificar o comportamento de permanência na fila.

Num estudo experimental, Mann (1977) instruiu confederados a se posicionarem em fila num ponto de ônibus em Israel, ambiente onde não havia a cultura da fila única, de maneira que fosse um estímulo para a organização de filas por quem chegasse ao local. Havia-se verificado que os usuários mantinham a ordem de acesso ao ônibus mesmo sem permanecer em uma fila propriamente dita. Assim, filas experimentais com até quatro confederados não geraram diferenças significativas na adesão de usuários que subsequentemente chegavam ao local. Entretanto, obteve-se uma adesão de quase $60 \%$ dos que chegaram após a formação da fila experimental com seis confederados e $83 \%$ quando a fila experimental era formada por oito confederados.

Argumentando que a fila deveria ser tratada como um sistema social, Milgram et al. (1986) realizaram experimento no qual confederados furaram filas para comprar bilhetes de metrô na cidade de Nova Iorque, dizendo apenas "com licença, eu gostaria de entrar aqui". Observaram tanto a reação do membro da fila no ponto de intrusão quanto a das demais pessoas na fila. O objetivo era demonstrar que o custo de uma intrusão está mais relacionado a um ultraje moral do que a prejuízos individuais por perda de tempo com aumento da espera. Entrando na terceira posição de filas com média de seis usuários observaram-se reações físicas aos intrusos em $10 \%$ dos casos e reações verbais em $22 \%$ dos casos, além de reações indiretas, tais como olhares hostis, gestos ou comentários com outros usuários na fila. Iglesias (2007) replicou esse experimento numa rodoviária no Brasil, em filas de 20 usuários em média, ocupando a $10^{\mathrm{a}}$ posição, sem oferecer qualquer justificativa aos usuários no ponto de intrusão. A frequência de reações diretas foi ainda menor que no estudo de Milgram et al. (1986), não se verificando diferenças significativas em função do sexo do intruso, da vítima ou do tamanho diferenciado das filas.

Schmitt, Dubé e Leclerc (1992) argumentaram que não foi possível diferenciar entre os custos morais e individuais no estudo de Milgram et al. (1986), razão pela qual estudaram a reação a diferentes justificativas para a intrusão na fila. Verificaram que intrusões justificadas por ação de um funcionário ou por uma "falha no sistema" geraram reações menores do que as de intrusos sem justificativa, mantendo-se o mesmo tempo de atraso em todas as condições. Schmitt et al. (1992), utilizaram cenários fictícios, além de uma replicação do experimento de Milgram et al. (1986), encontrando resultados similares. Esse foi o último experimento realizado com filas espontâneas em campo e publicado na literatura psicossocial de que temos conhecimento. Trabalhos posteriores utilizaram simulações no computador, cenários, escalas e surveys. Desde então, estudos sobre filas tornaram-se menos sistemáticos na psicologia social, sendo que atualmente se observa certa predominância de estudos em marketing, comportamento do consumidor e administração.

Nas áreas aplicadas o capítulo de Maister (1985) propondo uma "psicologia das filas de espera" 
provavelmente é a referência mais citada nos trabalhos sobre filas. Nele o autor apontou a falta de estudos com os fenômenos subjetivos relacionados à experiência da espera em filas, formulando uma série de hipóteses testáveis empiricamente e apontando direções de pesquisa sobre estimativas do tempo de espera e avaliação do serviço pelos usuários. Embora quase todas as proposições de Maister sejam da esfera da psicologia social, os estudos subsequentes pouco recorreram a essa área para fundamentação.

Não obstante, mais recentemente tem-se verificado interesse das áreas aplicadas em fundamentar teórica e metodologicamente a diversidade de estudos sobre filas, sendo que alguns autores reconhecem a necessidade de retornar à psicologia social para dar integração às pesquisas (Durrande-Moreau \& Usunier, 1999). Goodwin, Smith e Verhage (1991) ressaltam criticamente que a pesquisa sobre filas chega ao ponto de ser ateórica. A literatura mostra que grande parte das pesquisas sobre filas são estudos de caso ou carecem de validade externa, comprometendo o valor de seus resultados sobre um fenômeno que, por definição, depende do ambiente imediato, de normas sociais e da cultura.

\section{A FILA COMO TEMA E PALCO DE ESTUDOS DA PSICOLOGIA SOCIAL}

Diante desta revisão da literatura sobre a fila de espera, ainda que apareçam pesquisas empíricas, é possível chegar à conclusão de que o tema não somente está sendo pouco estudado, mas também tem considerável potencial para investigações na área de psicologia social. A seguir, mencionamos duas vertentes deste potencial: estudos sobre teorias do comportamento humano no contexto urbano e o uso da fila como palco de estudos sobre outros temas.

Anteriormente citamos a preocupação de Goodwin et al. (1991) sobre a natureza ateórica das pesquisas sobre fila. Destaca-se, porém, que, por ser um fenômeno social ubíquo, tanto de fácil observação quanto de fácil manipulação experimental, a fila oferece possibilidades consideráveis de estudos críticos acerca de diferentes teorias no âmbito da psicologia social, independentemente de suas aplicações imediatas. Atualmente, estudos sobre filas visam mais à solução de problemas gerenciais de características indesejáveis das filas, pelo papel central que ocupam na avaliação do serviço pelo consumidor. Objetivam intervenções práticas, como na qualidade do ambiente de espera, o efeito de seus atrativos, avisos sobre o andamento da fila, relógios, espelhos e outros recursos. Por outro lado, esses estudos revelam pouco sobre a dinâmica de fenômenos interpessoais, objeto tradicional de pesquisa em psicologia social. A título de exemplo do que pode ser desenvolvido, destaca-se a possibilidade de se estudar teorias de justiça, tanto distributiva quanto processual, atribuição de causalidade, agressividade, difusão da responsabilidade, diferenças interculturais e diversos fenômenos de cognição social (Iglesias \& Günther, 2007).

Finalmente, quanto ao potencial de filas como palco de estudos, cabe alertar que filas constituem aglomerações de pessoas, razão pela qual constituem uma excelente oportunidade para realizar entrevistas e até aplicar breves questionários, desde que, obviamente, se tome cuidado com a possível interferência das demais pessoas da fila.

Ao levar em conta que os membros de uma fila têm um objetivo que as une, cabe ter cuidado com a representatividade de uma pesquisa feita com essa população, embora Ellsworth e Gonzalez (2003) argumentem que pessoas em fila podem ser usadas nas pesquisas para se conseguirem amostras mais representativas da população, já que a psicologia social costuma receber críticas por recrutar, em sua maioria, estudantes universitários ou outras amostras convenientes. Considerando-se a complexidade do fenômeno, a diversidade no tratamento teóricoconceitual e a multiplicidade de abordagens metodológicas disponíveis, tanto qualitativas quanto quantitativas, filas podem servir como laboratórios naturais de psicologia (Sommer, 1977). Desta maneira, podem ser desenvolvidas pesquisas que tenham não necessariamente como foco a fila per se, mas qualquer comportamento que nela possa surgir, considerando-se ainda as evidências de que pessoas em filas colaboram bem com pesquisadores e não percebem a abordagem como incômoda ou como perda de tempo (Durrande-Moreau \& Usunier, 1999).

Esta análise psicossocial das filas procurou criar insumos para estender ao contexto brasileiro o projeto iniciado por Mann (1969) de que as filas sejam analisadas como sistemas sociais, regidas por normas e papéis definidos, sujeitas a valores, à cultura e ao ambiente imediato. Desenvolve, assim, o projeto de fazer uma psicologia social e ambiental das filas (Maister, 1985; Mann, 1970), por serem elas, fundamentalmente, problemas de comportamento social, indissociáveis da dinâmica do espaço urbano onde ocorrem. Isso amplia as possibilidades desse objeto de estudo na psicologia como um todo, mas também significa estender essa visão a outras áreas que investigam filas de espera, 
principalmente aquelas envolvidas com as trocas sociais.

\section{REFERÊNCIAS}

Adamatzky, A. (2005). Dynamics of crowd-minds: Patterns of irrationality in emotions, beliefs and actions. Toh Tuck Link, Singapura: World Scientific.

Aronson, E., Wilson, T. D., \& Akert, R. M. (2003). Psicologia social. Rio de janeiro: LTC Editora.

Baron, R. A., \& Byrne, D. (2003). Social psychology. Boston: Allyn $\&$ Bacon.

Beauvois, J. -L., Mugny, G. \& Oberlé, D. (Eds). (1995). La psychologie sociale: Vol. 1. Relations humaines, groupes et influence sociale. Grenoble: PUB.

Brown, R. W. (1954). Mass phenomena. In G. Lindzey (Ed.), Handbook of social psychology (Vol 2, pp. 833 876). Cambridge, M.A.: Addison Wesley.

Cogan, S. (1998). Gerenciando as percepções nas filas de espera._Rio de Janeiro: Qualitymark.

Cooper, R. (1981). Introduction to queuing theory. North Holland: Elsevier.

Cullis, J. G. \& Jones, P. R. (1985). National health service waiting lists: A discussion of competing explanations and a policy proposal. Journal of Health Economics, 4, 119-135.

Durrande-Moreau, A., \& Usunier, J. C. (1999). Time stiles and the waiting experience: An exploratory study. Journal of Service Research, 2, 173-186.

Ellsworth, P. C. \& Gonzales, R. (2003). Questions and comparisons: Methods of research in social psychology. In M. A. Hogg \& J. Cooper (Eds.), The Sage Handbook of Social Psychology (pp. 2442). Thousand Oaks, CA: Sage Publications.

Ferreira, A. B. H. (1986). Novo dicionário da Língua Portuguesa. Rio de Janeiro: Nova Fronteira.

Gilbert, D. T., Fiske, S. T., \& Lindzey, G. (Eds.) (1998). The handbook of social psychology (4th ed.). New York: McGrawHill.

Goodwin, C., Smith, K. L., \& Verhage, B. J. (1991). An equity model of consumer response to waiting time. Journal of Consumer Satisfaction, Dissatisfaction, and Complaining Behavior, 4, 129138.

Iglesias, F., \& Günther, H. (2007). Normas, justiça, atribuição e poder: Uma revisão e agenda de pesquisa sobre filas de espera. Estudos de Psicologia,12(1), 3-11.

Iglesias, F., Günther, H., Gouveia, B., Leitão, C., Presotti, L., Bandeira, R., Nogueira, R., Cartaya, S. \& Torres, C. V. (2004). Dissonância cognitiva em filas: Efeito da posição do usuário na estimativa do número de pessoas à frente. [Resumo]. In Sociedade Brasileira de Psicologia (Org.), Resumos de comunicações científicas. XXXIV Reunião Anual de Psicologia. Ribeirão Preto: SBP.

Iglesias, F. (2007). Comportamentos em filas de espera: Uma abordagem multimétodos. Tese de doutorado, Universidade de Brasília, Brasília.

Jones, M. T., O'Berski, A. M. \& Tom, G. (1980). Quickening the queue in grocery stores. Interfaces, 10, 90 .
Kostecki, M. (1996). Waiting lines as a marketing issue. European Management Journal, 14, 295-303.

Larson, R. C. \& Odoni, A. R. (1981). Urban operations research. Englewood Cliffs: Prentice-Hall.

Le Bon, G. (1954). Psicologia das multidões. Rio de Janeiro: F. Briguet \& Cia. (Originalmente publicado em 1895)

Levine, R. (1997)._A geography of time._New_York: Basic Books.

Maister, D. (1985). The psychology of waiting lines. In J. A. Czepiel, R. Solomon \& C. F. Suprenant (Eds.), The service encounter: Managing employee/customer interaction in service businesses (pp.113-123). Lexington: Lexington Books.

Mann, L. (1969). Queue culture: The waiting line as a social system. American Journal of Sociology, 75, 340-354.

Mann, L. (1970). The social psychology of waiting lines. American Scientist, 58, 390-398.

Mann, L. (1977). The effect of stimulus queues on queue-joining behavior. Journal of Personality and Social Psychology, 35, 437442.

Mann, L. \& Taylor, K. F. (1969). Queue counting: The effects of motives upon estimates of numbers in waiting lines. Journal of Personality and Social Psychology, 12, 95-103.

Milgram, S. (1970). The experience of living in cities: Adaptations to urban overload create characteristic qualities of city life that can be measured. Science, 167, 1461-1468.

Milgram, S. \& Toch, H. (1969). Collective behavior: Crowds and social movements. In G. Lindzey \& E. Aronson (Eds.) The handbook of social psychology (2nd ed., vol. 4, pp. 507-610). Reading, MA: Addison-Wesley.

Milgram, S., Liberty, H. J., Toledo, R. \& Wackenhut, J. (1986). Response to intrusion into waiting lines. Journal of Personality and Social Psychology, 51, 683-689.

Myers, D. J. (2000). Psicologia social. Rio de janeiro: LTC Editora.

Rafaeli, A., Barron, G. \& Haber, K. (2002). The effects of queue structure on attitudes. Journal of Service Research, 5, 125-139.

Rodrigues, A., Assmar, E. M. L. \& Jablonski, B. (2000). Psicologia social. Petrópolis: Vozes.

Rothkopf, M. H. \& Rech, P. (1987). Perspectives on queues: Combining queues is not always beneficial. Operations Research, $35,906-909$.

Rozestraten, R. J. A. (1988). Psicologia do trânsito: Conceitos e processos básicos. São Paulo: EPU/EDUSP.

Schmitt, B. H., Dubé, L. \& Leclerc, F. (1992). Intrusions into waiting lines: Does the queue constitute a social system? Journal of Personality and Social Psychology, 63, 806-815.

Schwartz, B. (1975). Queuing and waiting: Studies in the social organization of access and delay. Chicago: University of Chicago Press.

Schwartz, B. (1978). Queues, priorities, and social process. Social Psychology, 41, 3-12.

Simmel, G. (1987). A metrópole e a vida mental. In G. O. Velho (Org.). O fenômeno urbano (pp.11-25). Rio de Janeiro: Guanabara. (Trabalho original publicado em 1903)

Smith, E. R. \& Mackie, D. M. (1995). Social psychology. New York: Worth Publishers.

Sommer, R. (1977). Toward a psychology of natural behavior. APA Monitor, 8(1), 1-7. 
Surowiecki, J. (2004). The wisdom of crowds. New York: Doubleday.

Vala, J. \& Monteiro, M. B. (2004). Psicologia social. Lisboa: Fundação Calouste Gulbenkian.

Zohar, E., Mandelbaum, A., \& Shimkin, N. (2002). Adaptive behavior of impatient customers in tele-queues: Theory and empirical

Recebido em 28/09/07 support. Management Science, 48, 566-583.

Aceito em 23/10/08

Endereço para correspondência :

Fabio Iglesias, Universidade de Brasília, ICC Sul, Instituto de Psicologia, Laboratório de Psicologia Ambiental, CEP 70910-900, Brasília-DF, Brasil. E-mail: iglesias@unb.br 